\title{
La posición del Estado ante los otros actores
}

\section{Michel Crozier *}

\section{La obsolescencia de nuestras ideas nos impide comprender una realidad profundamente diferente}

El Estado nacional ya no tiene frente a sí los mismos actores dispersos, relativamente previsibles que podía dominar fácilmente. El Estado tiene frente a sí a actores más numerosos, más heterogéneos, más independientes, que son capaces de aliarse entre ellos y de funcionar sin respetar fronteras. Estos actores disponen de un gran poder de negociación. Logran penetrar en el Estado e influenciarlo más de lo que ellos mismos son influenciados.

Al mismo tiempo, el Estado nacional, que consideramos en Francia como necesariamente unitario, se ha diversificado extraordinariamente hasta el punto que hablar de un solo actor en este conglomerado de actividades diversas aparece cada vez más como una ficción, puede que cómoda, pero que de hecho falsea su significado.

En definitiva, el juego entre actores de la vida económica y de la vida política se ha transformado radicalmente a causa de la evolución acelerada de las sociedades modernas postindustriales. Las relaciones de poder, los medios para lograr influencia, las comunicaciones y los problemas ya no son los mismos .

A pesar de ello, seguimos discutiendo sobre el papel del Estado y su funcionamiento frente a los otros actores como si nos encontrásemos en la situación del Estado nacional de principios de siglo. Un Estado capaz de conservar fuertemente el monopolio del interés general en su territorio, capaz de definirlo como único y de imponer las reglas del juego y las condiciones de negociación a todos los actores bajo su jurisdicción, capaz también y sobre todo de representar, o más bien de hacer derivar de este hecho el interés general de todos los actores de la nación, frente a los otros actores nacionales -los otros Estados, y del pequeño número de actores supranacionales, relativamente impotentes, de la época.

Los problemas que derivan del desarrollo político de Europa y de los múltiples órganos de cooperación internacionales, por una parte, y de la mundialización de los cambios y de la explosión de las comunicaciones, de otra, son totalmente insolubles si seguimos razonando a través de esta visión tradicional. Los conceptos clásicos del Estado son obsoletos. A pesar de todo, su influencia, aún considerable, nos impide encontrar soluciones aceptables para los problemas que no llegamos ni siquiera a aprehender, faltos de categorías mentales adaptadas a la nueva realidad.

Mientras sigamos pensando que la unidad es en sí misma superior a la diversidad, que en la República una y indivisible, sólo el Estado unitario debe encarnar el interés general y que este interés general, definido por los órganos legales del Estado a partir del voto democrático que basta para legitimarlos, debe imponerse a todos los ciudadanos y sobre todas las actividades, tendremos una actitud ambivalente frente a toda superación de esos límites y en primer lugar frente a Europa. Permaneceremos divididos entre la esperanza de una mayor fuerza del concepto de humanidad y el miedo a perder nuestra identidad demasiado ligada a este Estado nacional, que constituye la clave de bóveda de todas nuestras identidades colectivas. Es imposible tratar razonablemente las simbiosis cooperativas y conflictuales que constituyen la trama de la vida de nuestras sociedades hipercomplejas. Obsesionados por la soberanía, imaginamos divisiones 
complicadas que estremecen a todo el mundo (para empezar a nosotros mismos), y que acabaremos no respetando. Acabaremos siendo llamados hipócritas y no llegaremos a comprender la evolución acelerada que se desarrolla ante nosotros.

Vivimos esta evolución y estas conmociones no solamente en las relaciones del Estado-nación con los otros Estados-nación, sino al interior mismo de cada Estado-nación. El desarrollo de la Europa comunitaria nos hace tomar conciencia de todos estos hechos. Pero atribuir nuestras dificultades a Europa o a las malas interpretaciones que hemos hecho sobre lo que representa Europa. El problema es mucho más profundo. Se debe al cambio acelerado del mundo. La presión que representa la nueva realidad europea no hace sino acentuar esa aceleración.

Si queremos reflexionar seriamente sobre el funcionamiento del Estado frente a los otros actores en el contexto y el marco europeo, debemos necesariamente superar los límites de la Europa actual y de nuestros problemas. En primer lugar, debemos tomar consciencia de la realidad del mundo postindustrial e interrogarnos sobre el concepto mismo de Estado y sobre las nuevas condiciones de las relaciones entre actores en un mundo diferente.

\section{¿En qué afecta el cambio del mundo a la realidad del Estado y sus relaciones con los demás actores?}

En cincuenta años el cambio del mundo ha sido mucho más extraordinario de lo que nosotros creemos.

La multiplicación del nivel de vida por dos, tres o hasta cinco años en los países más desarrollados ha provocado seguramente más problemas que soluciones. Ello no nos ha traído ni la igualdad ni la felicidad, pero tampoco se trata de una simple patraña estadística, sino que ha transformado las relaciones humanas: más riqueza es igual a más libertad de escoger, a más posibilidades individuales, a más independencia de los individuos en relación a las estructuras.

El crecimiento vertiginoso de los cambios ha multiplicado las interacciones entre los individuos en el interior de una sociedad y de una sociedad a otra. La complejidad ha sobrepasado los umbrales a partir de los cuales los medios de control o de dominio tradicionales han perdido toda influencia y hasta todo sentido.

La explosión aún mas vertiginosa de las comunicaciones ha trastornado las relaciones de los individuos en la sociedad, en los Estados y en las estructuras en general.

El viejo mundo fundado sobre la distancia, el tiempo, las barreras y las jerarquías, a pesar de las apariencias, ya no existe. Sin duda alguna, las instituciones subsisten, las reglas permane- cen, y hasta crecen desmesuradamente, pero no tienen la misma función ni el mismo impacto. Cuando ocurre un hecho tan inesperado como lógico como es el hundimiento del sistema totalitario de los países del Este, la distancia entre sociedades nos parece casi impensable. Nada se puede resistir al torbellino que nos arrastra. Aquellos a los que un régimen conservador había petrificado en lógicas anteriores tienen un atraso que se nos presenta como insalvable.

¿Qué hay de profundo en esta aceleración de la evolución reciente? ¿Cuáles son las nuevas condiciones que afectan al papel de los actores? ¿Qué problemas se derivan para el Estado de este hecho? Para comprenderlo deben intentarse analizar las tendencias que dominan la evolución de nuestras sociedades y sus consecuencias. Distinguiría cuatro, aun a riesgo de simplificar: 1) la evolución hacia la complejidad; 2) el aumento de la libertad de elección de los individuos; 3) la transformación de la lógica económica, y 4) la mediatización de las relaciones humanas.

\section{- La evolución bacia la complejidad}

Los desarrollos tecnológicos consecuencia del desarrollo científico conducen hacia la racionalización y la simplificación de muchas operaciones. Finalmente se ha reconocido que su impacto general comporta una complejidad y una interdependencia cada vez mayor de nuestras actividades. Puede que el mundo se haya "desencantado", pero no por ello se ha convertido ni en más simple ni en más claro. Crear un nuevo producto necesita la colaboración de un número cada vez más grande de especialistas de lógicas y referentes diferentes. La estrecha visión racional, que basa su posible cooperación en el puro cálculo se va revelando como falsa. La capacidad de cooperación de los grupos humanos es, en muchos casos, más importante que la visión individual de sus miembros. El problema es mucho más impresionante en materia económica y financiera. El aumento de intercambios se traduce por una interacción de más actores con otros actores, lo que podría conducir a una progresión geométrica imposible de dominar por cerebros humanos. De hecho, esto significa que hay nuevas estructuras de mediación que van surgiendo en lugar de las antiguas estructuras que la aceleración de la evolución ha hecho estallar. Pero estamos en crisis perpetua. Y aún con ambiciones más modestas que antaño, no llegamos a repensar sistemas de regulación satisfactorios.

En el mundo de la decisión política y social y en materia ecológica surgen dificultades considerables de otra naturaleza. La disminución de las barreras sociales, el acceso más fácil de los grupos más diversos a las instancias de decisión, la toma de conciencia del hecho del progreso de los conocimientos, las consecuencias secundarias de toda decisión colectiva, se vuelven mucho más complejas y difíciles.

De hecho, el Estado jerárquico, burocrático, ya no puede responder a los problemas derivados del aumento de la complejidad. Se encuentra con las mismas contradicciones que las grandes empresas que no llegan a dominar los conjuntos demasiado grandes y pierden el contacto con la realidad. 
De todo lo cual se deriva que el concepto de Estado unitario, indivisible, representante del interés general, comporta burocracia y jerarquía. Y las tareas que se le han atribuido y que se le han dado, son tan complejas que son imposibles de dominar desde el vértice. Las autoridades públicas deben cambiar de lógica para que puedan abrirse suficientemente a la diversidad, para poder responder a la creciente complejidad.

\section{- La tendencia irresistible al incremento de la libertad indivi- dual}

Hemos razonado demasiado a partir de una visión estrechamente política de la libertad. Obsesionados por la preponderancia (que luego ha resultado provisional) de las grandes máquinas burocráticas, corporaciones económicas y corporativismos, hemos subestimado el profundo movimiento de nuestras sociedades hacia la libertad individual.

Libertad precaria, insuficiente, que por contraste resalta el carácter escandaloso de las limitaciones que persisten, pero libertad irresistible que afecta a todas las sociedades que estén suficientemente desarrolladas. Libertad que no está a merced de un accidente político o de un cambio de moda ideológico, ya que no es consecuencia de una elección política o ideológica provocada por ella, sino que su origen está en la transformación de las condiciones mismas del desarrollo económico y social.

La riqueza individual, la complejidad de las interacciones colectivas y la facilidad de comunicación dan a un individuo post-moderno normal, posibilidades de afirmación y de libertad de elección desconocidas hasta ahora. A más libertad, mejores condiciones de negociación con todos los poderes económicos, políticos, sociales y familiares. La naturaleza del juego entre gobernantes y gobernados, superiores y subordinados, hombres y mujeres, padres e hijos, se modifica.

Cuando se examinan las consecuencias del desarrollo de esta libertad universal que, hasta ahora, aunque haya acarreado muchos problemas y angustias, nunca ha generado más que una demanda suplementaria, vemos que está ligada profundamente al desarrollo de la complejidad, que contribuye a generar y que al mismo tiempo, le hace posible afirmarse. Esto es lo que hace irresistible al movimiento. Ninguna sociedad, ningún gobierno puede oponerse mucho tiempo si no quiere condenarse al subdesarrollo.

Se constata que este movimiento cuestiona el Estado burocrático nacional, que se encuentra delante de un dilema infranqueable. Por un lado, se le pide dominar una complejidad que no puede llegar a abarcar. Por otro, los medios tradicionales que había desarrollado para reducir y controlar esta complejidad son cada vez menos productivos ya que los individuos, ya sea en política o en economía, tienen con su libertad los medios para no obedecer. No es la presión europea la que impide al gobierno francés controlar seriamente el sistema de cambios, sino que es el desarrollo de las comunicaciones y de los intercambios.

Durante mucho tiempo, la fuerza del Estado estaba ligada a la extensión de sus poderes y a la posibilidad que daba la relativa confusión de hacer presión al mismo tiempo sobre los indivi- duos y sobre los grupos. Hemos pasado a un sistema de relaciones dominado cada vez más por mecanismos inversos. Cuanto más poder, más vulnerabilidad frente a las presiones y menos capacidad de decidir y de dominar la complejidad. Cada vez aparece como más indispensable cambiar de lógica.

\section{- La lógica del desarrollo económico y social esta cambian- do}

Esta necesidad de cambio de lógica, dominada aún por los antiguos conceptos de Estado guardián del interés general, tiene muchos problemas para asentarse en el entorno público. Pero cada vez está más presente en la esfera de las actividades económicas y técnicas, cuyo impacto sobre el juego del Estado se acrecienta.

Estamos pasando de una lógica de sociedad industrial clásica basada en el ciclo de producción masiva / consumo masivo, a una lógica totalmente diferente basada en la alta tecnología y los servicios. La lógica clásica hace de la racionalización el motor esencial del progreso. Gracias a la racionalización se puede iniciar y desarrollar el ciclo: racionalización de la producción que permite producir más barato, vender más, producir en grandes series; racionalización del consumo que al estandarizarse permite mantener el ciclo. Claro está que detrás de la racionalización hay innovación y desarrollo tecnológico. Pero no es aquí donde se crea la diferencia. En la sociedad postindustrial los motores del desarrollo son la innovación tecnológica y la innovación en los servicios. Aquellos que no estén atentos a ello, y continúen dando absoluta preeminencia a la racionalización, van a quedar rápidamente condenados a la decadencia.

El Estado burocrático ha tenido éxito, hasta cierto punto, en ese mundo de la racionalización, pero desde hace mucho tiempo, lo que gana reglamentando y estandarizando, no puede en absoluto compararse con lo que hace perder a la sociedad asfixiando en iniciativas. El problema del desarrollo, de aqui en adelante, lo encontramos mucho más en el descubrimiento y en la generación de recursos que en la racionalización de su utilización.

Este razonamiento es precisa y especialmente válido para todos los servicios sofisticados que asegura actualmente el Estado, no únicamente la educación, la sanidad y la investigación sino también la gestión de los temas sociales, la regulación de las actividades económicas y, de forma más general, la promoción del interés general.

La visión estrecha de la gestión tomada en préstamo del management clásico de la sociedad postindustrial es particularmente inadecuado. De la misma manera que ha podido y aún puede, con ciertos límites, contribuir a mejorar los resultados de ciertas actividades de producción como las telecomunicaciones y los ferrocarriles, que por otra parte en algunos países están privatizados, sus limitaciones tienden a esterilizar la adaptación a la sociedad moderna de las actividades más indispensables, como la policía, la justicia o las actividades de regulación económica. 


\section{- La mediatización de las relaciones bumanas}

La explosión de las comunicaciones ya ha causado trastornos en las relaciones de los individuos con la colectividad, no solamente en la colectividad en su conjunto, sino en todas las colectividades, es decir, en todos los grupos humanos.

Las relaciones de poder no pueden ser las mismas cuando los medios modernos permiten la inmediatez de la comunicación y es imposible mantener el secreto.

Manipular la opinión es posible. Se ha usado y abusado de ello. Pero los manipuladores son cada vez más manipulados por un sistema sobre el cual todo el mundo puede influir, pero que ya nadie domina.

Esta explosión constituye una de las dimensiones de la evolución hacia una mayor libertad y complejidad. Pero al mismo tiempo crea una gran cantidad de problemas y dificultades que cuestionan no solamente las prácticas, sino también la lógica del Estado tradicional.

¿Cómo puede actuar el Estado de forma razonable? La mediatización de la política le hace muy vulnerable a las presiones de los actores, que pueden manipular la opinión en los momentos clave de esa vulnerabilidad. ¿Cómo podemos librarnos de la lógica infernal de la manipulación contradictoria de las emociones superficiales, lógica que amenaza con oscurecer todas las opciones colectivas hasta el punto de llegar a paralizar toda acción, obligando al político a limitarse a aquellas decisiones de carácter más mediático?

Una comprensión más profunda de estos fenómenos, un cierto "descompromiso" del Estado, abandonando los dominios más vulnerables, una inversión mucho más intensa en conocimiento y la difusión de los problemas realmente vividos, pueden hacer posible un cambio de lógica a medio plazo: partes importantes de las decisiones deberán ser dejadas a la lógica autorreguladora de profesionales y de ámbitos locales, el papel de las autoridades públicas se convierte cada vez más en un papel de garante.

\section{La concepción del interés general está a punto de cambiar}

La relación del Estado con los otros actores se conceptualizaba claramente en otros tiempos alrededor del interés general. El Estado encarnaba el interés general frente a los intereses particulares. Era él quien debía decidir siempre en último caso. Escuchaba a las partes, decidía y hacía aplicar sus decisiones. Lo hacía a través de sus órganos legales investidos de esa misión por el sufragio universal. Estaba protegido en nuestros países democráticos contra los riesgos del totalitarismo y de la corrupción, por el control que ejercían los elegidos, por la intervención de órganos de control interno, por la existencia de tribunales independientes y por la sumisión cultural a estrictas reglas éti- cas. Ciertamente, la realidad no respondía totalmente al modelo ideal. Pero, en gran medida, este sistema de check and balances funcionaba bastante bien.

En un Estado particularmente unitario como el Estado francés tradicional, el interés general era una verdadera religión. Cubría todas las actividades del Estado, hasta las más anodinas. En nombre del interés general, las instituciones estatales, administraciones, instituciones públicas y hasta empresas nacionales, disponían de un poder de negociación y de influencia considerable sobre su entorno y en consecuencia sobre la sociedad en su conjunto. Este poder no era excesivo en la medida en que el Estado estaba aún poco extendido y que las actividades de la sociedad civil se basaban en un alto nivel de autorregulación.

El crecimiento extraordinario de las actividades del Estado, que ha acompañado el desarrollo de la riqueza y de la complejidad de la sociedad, ha hecho estallar este sistema.

En primer lugar, la ficción del Estado como actor único, jerarquizando y coordinando todas las actividades que emanan del interés general, se convierte en insostenible. La confusión entre la sociedad civil y el Estado tiende a convertir todas las actividades en el interior de una sociedad en susceptibles de ser objeto de intervenciones públicas en nombre del interés general. Cuanto más se extienden sus competencias, más aumentan las presiones para influir en su orientación y se hacen más complejas, con lo que es más difícil determinar su curso y hacerlo aceptar.

Los órganos del Estado se han hecho mucho más complejos y se han diversificado mucho. Es imposible integrarlos directamente de manera oficial y clara. Por eso, el conjunto de organismos estatales es tan confuso e irresponsable como inmenso. Esta integración se intenta hacer en la cúspide a través de la intervención financiera, basándose en una lógica de coherencia a corto término. Se hace casi de forma clandestina, mediante un criterio de racionalidad a muy corto término. Por abajo, es muchas veces el actor exterior el que logra integrar para sí las lógicas contradictorias de las diferentes feudalidades administrativas incapaces de coordinarse.

En definitiva, la utilización del poder que da a un actor estatal la posibilidad de utilizar los enormes medios públicos de que dispone, gracias al control que ejerce sobre las actividades más diversas -finanzas, tecnología, gestión, servicios directos- aparece cada vez más exorbitante, ya que permite ejercer una especie de chantaje sobre los partenaires exteriores. Al mismo tiempo, se vuelve más vulnerable en la medida que, al hacer demasiada presión sobre el partenaire, acaba por permitirle que él también ejerza presión. De esta forma, las Administraciones acaban convirtiéndose a menudo en prisioneras de los sectores a los que ejercen una influencia excesiva.

Frente a la fragmentación teóricamente individual del actor de antaño, los partenaires del Estado se han transformado. En primer lugar, son mucho más numerosos porque la sociedad, mucho más compleja, ha propiciado la creación de un mayor número de grupos, y asimismo porque la acción del Estado ha provocado el aumento de los sujetos de derecho. En segundo lugar, porque la disminución de las barreras sociales y la 
explosión de las comunicaciones han hecho imposible prohibir el acceso de la mayoría a las decisiones. Estos actores más numerosos son extraordinariamente heterogéneos. No se puede contar ni con su estabilidad ni con su fiabilidad. El aumento de la libertad individual disminuye la presión de cada grupo sobre cada uno de sus miembros. De manera general se constata un debilitamiento de las estructuras capaces de negociar y de comprometerse. De ahí la impresión de labilidad general y de movernos en el reino de la apariencia, en el cual la mediática acaba convirtiéndose en un punto de anclaje indispensable

$Y$ sin embargo se crean equilibrios y se ejerce el poder regulador. El sistema, bien o mal, funciona. El problema esencial, si se quiere actuar al interior de este sistema y aún más si se quiere reformar, es el de conocer el sistema en su práctica real. Se vive demasiado en un mundo de apariencias a causa de la insistencia jurídica y política de empezar a partir de lo que debería ser y no de lo que es. Desde luego, sabemos que la realidad es diferente, pero la intentamos captar sólo desde una visión crítica de la referencia normativa.

Este conocimiento de la realidad, absolutamente indispensable, es tan difícil de establecer que, frente a las normas generales que deberían aplicarse debido a la universalidad de los principios a los que se refieren, lo que predomina son las regulaciones concretas y diversas que gobiernan las prácticas según los parámetros particulares de cada situación. Por otro lado, estas regulaciones solamente tienen sentido en el interior del sistema de relaciones en el cual el juego del Estado puede ser más o menos estructurador. Sólo podremos llegar a entender algo de cómo funcionan las cosas, desde una lógica sistémica, y para ello se impone una conversión conceptual que la mayoría de los responsables no están preparados para llevar a cabo.

La acción del Estado en el sistema de relaciones industriales frente a los actores sindicales y nacionales no tiene nada que ver con su intervención en materia de ayuda a las PME o en materia de innovación. En ejemplos como éstos, los actores públicos nunca son únicos, los actores "sociales" siempre son múltiples, relativamente poco representativos, y el juego siempre da más vueltas alrededor de la apariencia mediática de la decisión producida que de su importancia real. Estos sistemas cambian relativamente rápido. Algunos siguen ritmos relativamente regulares de ascensión y de decadencia, de desaparición y de refundación. El problema del acceso es a menudo, pero no siempre, decisivo. La dificultad más grande generalmente es la imposibilidad de encontrar una solución intermedia aceptable entre el riesgo de colisión que conlleva una excesiva simbiosis con los actores y el riesgo de ignorancia, de incompetencia y de arbitrariedad que puede provocar una excesiva distancia con esos mismos actores.

Ese sistema de relaciones tradicional se complica aún más por la aparición de actores externos a la sociedad francesa. Los actores transnacionales pueden reforzar, pero también contradecir y desequilibrar a los actores nacionales. Es fácil, en abstracto, criticar a los lobbys en general y a los lobbys de Bruselas en par- ticular, pero un Estado excesivamente desarrollado, rodeado por todas partes y en una situación de debilidad, a menudo lo encontramos prisionero de esos lobbys que lo penetran y le permiten hasta cierto punto salvaguardar la apariencia de potencia que quiere mantener.

La reflexión de los politólogos durante los años setenta estuvo marcada por el descubrimiento de los sistemas corporativistas transnacionales que parecían ser todopoderosos frente a la debilidad y a la fragmentación de las autoridades públicas. Las grandes corporaciones americanas han salido mal paradas de los años ochenta, ante la sorpresa de todo el mundo, y los sistemas corporativos transnacionales se han asentado menos de lo que se temía. En revancha, la confusión y la imposibilidad de dominar la complejidad de los sistemas reales de decisión ha llevado a los protagonistas del debate sobre el control de los lobbys a una mayor modestia.

El cambio más grande que ha sufrido el Estado francés concierne a la naturaleza de sus relaciones de poder. Hasta hace veinte años, los estadistas estaban seguros de ser capaces de definir el interés general. Su problema era el de saber como hacerlo prevalecer. Por esto, utilizaban el mando directo cuando aún podían, pero cada vez era más raro y las presiones indirectas que podían ejercer gracias a su situación dominante en un gran número de actividades les permitía recompensar a los actores que seguían sus indicaciones y castigar a los que se resistían a ello.

Ese sistema se ha degradado cada vez más mientras que el problema se ha desplazado. Ya no se trata de hacer prevalecer el interés general sino de hacer emerger un consenso suficiente alrededor de una visión aceptable del interés general para los que tendrán que aplicarlo en sus decisiones. Hecho que implica no centrarse más sobre la decisión sino sobre los procesos de su elaboración, sobre su puesta en práctica y sobre los resultados que de ella se obtengan.

\section{¿Cómo asegurar la necesaria renovación de la actuación del Estado?}

La transformación de las condiciones generales en que se desarrollan las actividades humanas modifica radicalmente los sistemas de relaciones humanas en cuyo seno gobernantes y funcionarios tenían costumbre de actuar. No se pueden obtener resultados aceptables utilizando mecanismos del tipo jerárquico tradicional. El irresistible movimiento hacia la libertad no se da solamente en el Este a través de la ruptura de las barreras burocráticas. En Francia, como en todo el Occidente, ni los administrados ni los funcionarios obedecen como antes. Los responsables máximos ya no creen en las virtudes del sistema. Los antiguos mecanismos sobre los que se apoyaban son cada vez menos eficaces y no se conocen otros mecanismos. 
Tenemos que hacer frente a una crisis de legitimidad de orden burocrático al mismo tiempo que a una crisis de orden moral e intelectual en los servidores del Estado.

Para avanzar debemos ante todo trabajar para la renovación de las formas de intervención del Estado. No existe una respuesta simple pero sí algunas observaciones generales que pueden permitir definir el problema.

Primera observación: la revolución postindustrial no comporta la decadencia de las actividades públicas, que cada vez son más numerosas e indispensables sino más bien al contrario, la revolución postindustrial impone el cambio radical en la lógica de las actividades públicas y en el modo en que son ejercidas. La creciente complejidad de las actividades humanas, su interdependencia y la mayor libertad de los actores nos conducen a la decadencia del Estado en provecho de un gran mercado de regulaciones automáticas. La aceleración de los cambios conlleva a la vez una extensión extraordinaria de las prácticas de mercado y una necesidad paralela de regulaciones y de servicios de todo orden que solamente pueden ofrecer las autoridades públicas.

Si existe crisis es debido a una falta de organización colectiva y el Estado unitario es incapaz de asegurarla solamente con los instrumentos cada vez más inútiles de la jerarquía, o de sus prerrogativas monopolistas. La quiebra de los regímenes totalitarios, que se nos presenta a veces como caricatura, constituye un ejemplo a meditar

Para que el Estado pueda cumplir su rol en un contexto que sea radicalmente diferente, es necesario que invierta considerablemente en medios y necesita una gran perseverancia para elaborar nuevos medios de acción compatibles con la nueva naturaleza de las pautas de interacción público-privado.

Segunda observación: la lógica de la unidad de un Estado por encima de las reglas de la sociedad era utilizada hasta ahora por el Estado nacional como recurso de negociación para doblegar toda resistencia. Pero esta lógica no solamente ya no es aceptada sino que en la práctica es inoperante y debilita cada vez más nuestras instituciones.

La confusión mantenida de forma inteligente, que permite a los gobernantes y a los grandes e incluso a los pequeños administradores el intervenir en todos los ámbitos de la sociedad y en relación a todos los problemas, sirve a corto plazo a estos operadores, pero a largo plazo arruina la legitimidad del Estado hasta el punto de convertirlo totalmente en impotente.

En el mundo en que vivimos actualmente no podemos salirnos con la nuestra en contra de los ciudadanos, presionándolos, haciéndoles chantaje. Es apoyándonos en ellos, ayudándoles a comprender sus problemas y a tomar conciencia de que también ellos encarnan el interés general como podremos elaborar y mantener las regulaciones que la sociedad precisa.

Desde luego, algunas funciones de integración son necesarias. Pero lo que era la regla deberá ser la excepción. Las decisiones técnicas y administrativas deben ser juzgadas a partir de sus méritos propios y no porque el órgano de decisión que las toma dispone de un poder exorbitante que le confiere su simple pertenencia al Estado como representante único del interés general.

Es indispensable que la mayoría de servicios que garantizan el Estado o las autoridades públicas no puedan esconderse más bajo la capa protectora del poder público. Su privatización no es estrictamente necesaria. Puede ser útil que sean garantizadas por autoridades públicas neutras y que estén sometidas a reglas especiales. Pero ya no pueden formar parte del sistema tradicional de intervención del Estado y constituir instrumentos de poder potencial para el mismo.

Tercera observación: para poder ser más eficaz, dada la naturaleza de las relaciones de poder dominantes, el Estado nacional no solamente debe diferenciarse y especializarse sino que también debe de alguna manera de "descomprometerse".

En la visión tradicional de las relaciones de poder, era no sólo útil sino indispensable acumular funciones y confundirlas para extender cada vez más el campo de autoridad, sin por ello asumir las responsabilidades consiguientes.

En el marco de la nueva complejidad, cuantos más amplios poderes tenga la autoridad reguladora, más vulnerable será y menor capacidad tendrá para actuar. Este cambio de lógica implica que el Estado se retire eventualmente de ciertos campos en beneficio de las autoridades profesionales o territoriales y que, en otros campos, se descargue de funciones de gestión en beneficio de agencias autónomas en las que no está implicado. Esta retirada tendrá la inmensa ventaja de hacerlo menos vulnerable a las presiones y le permitirá consagrarse más serena y profesionalmente a las tareas de interés general y, más particularmente, a las tareas de regulación que son cada vez más cruciales.

Cuarta observación: la especialización del Estado en las tareas de regulación no debe llevar a que los funcionarios se arroguen el monopolio de la expertise convirtiéndose en los reguladores de la vida social y económica.

El ideal a desarrollar, difícil pero indispensable, es el de la autorregulación. Si las autoridades públicas y sus funcionarios al servicio de la sociedad son indispensables, sólo lo son en tanto y en cuanto ello permita llegar a elaborar regulaciones estables. Es indispensable un inmenso trabajo de conocimiento, de grupo, de emergencia de consenso, de experimentación, y si las autoridades públicas no son indispensables para garantizarlo, sí lo son al menos para hacerlo posible.

Quinta observación: la lógica jerárquica tradicional, que defiende que el poder no debe fragmentarse, debe dejar paso a la lógica de la corresponsabilidad. El poder debe fragmentarse y puede hacerlo si las condiciones en que se ejerza se organizan con esmero y respeto. En Europa actualmente hay una gran cantidad de sistemas de decisión político-administrativos. Dan a la acción colectiva un ámbito de actuación mucho más amplio y permiten dominar mejor el juego de la complejidad.

La Europa comunitaria, que ha sabido servirse con mucho provecho de la técnica de la colegialidad, nos ofrece una historia 
enriquecedora de experimentación natural. Su ejemplo se puede exportar perfectamente.

Generalmente, los problemas planteados por la construcción de Europa constituyen una gran oportunidad para experimentar nuevas formas de acción colectiva transportables a la organización interna de las sociedades nacionales.

Sexta observación: lo más importante para el éxito colectivo será cada vez más la interiorización del interés general, por parte de los actores privados, de objetivos y medios y no la obediencia a una idea de racionalidad externa definida por una autoridad superior.

El problema esencial es el saber propiciar el necesario consenso en la elaboración del concepto de interés general. La experiencia nos muestra que es alrededor del conocimiento de los problemas y no en torno a la discusión de las soluciones como podremos llegar al consenso. Se deberá hacer un gran esfuerzo para invertir la lógica tecnocrática centrada en las soluciones, para desarrollar los métodos que nos permitirán hacer surgir un consenso: la rehabilitación del trabajo de comisión, la organización de la colegialidad, la utilización del conocimiento; medios para tomar conciencia de los problemas desdramatizando las soluciones.

Séptima observación: inversión en el conocimiento de las realidades de la acción colectiva, formación en métodos de preparación de decisiones y de evaluación de resultados, el requerimiento a los miembros de la sociedad civil para animar las comisiones de reflexión y de análisis de los problemas, son todas cuestiones clave para asegurar la renovación del juego del Estado.

Las estructuras y las estrategias racionales no marcarán la diferencia, sino que será la práctica de los funcionarios la que la marcará, y la acción eficaz será la que conseguirá transformar la lógica.

Ya no se trata de dirigir directamente o indirectamente a los ciudadanos ni de hacerlos actuar sino de crear condiciones de reencuentro, de aportar los conocimientos, las herramientas y las experiencias para que aquellos a los que uno quiere ayudar puedan decidir por ellos misnos.
Traducción de Joaquim Colominas i FERRAN.

- Michel Crozier, es probablemente el especialista más conocido en teoría de la organización y no sólo en Francia. Sus célebres obras, le Phenomène Bureaucratique, o L'acteur et le Système (este último con Erhard Friedberg), le consagraron mundialmente. Entre sus muchas obras y aportaciones, destacaríamos la recientemente publicada, Etat Modeste, Etat Moderne (con traducción al espanol en FCE), en la que asume tesis que en el artículo que aquí presentamos amplia y matiza. Actualmente mantiene sus contactos con el Centro que creó de sociología de las organizacions integrado en el CNR francés, mientras ejerce de consejero científico de la sociedad SMG. 


\section{Muchos \\ de los directivos de la empresa española


Otros vienen para ponerse al día

- Master en Gestión Industrial.

- Master en Gestión de Calidad.

- Master en Ingeniería y Gestión Medioambiental.

- Master en Dirección de Empresas.

- Master en Gestión de las Comunicaciones y Tecnologías de la Información.

- Master en Ingeniería Medioambiental. Gestión del Agua.

SI:VII.I.A

Aicla. II"t. Isla de la ( "artujia H(1)I() Sevilla Tis.: (9.5) +2.375607 I:11: (1).5) +2.370 .82$





Mimisteriud de Industrial y lincrevia
Escuela de Organización Industrial

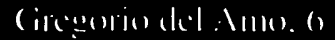

(cimdind l'miscrilamia)

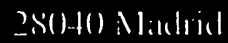

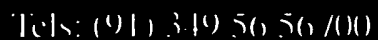

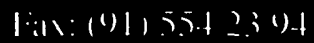

